\title{
Acute porphyrias and porphyric neuropathy
}

\author{
Doungporn Ruthirago MD, Parunyou Julayanont MD, Supannee Rassameehiran MD
}

\begin{abstract}
The porphyrias are a group of uncommon inherited metabolic disorders of heme biosynthesis. Acute porphyrias are specific types of porphyrias characterized by the presence of acute attacks that usually present with abdominal pain, psychiatric symptoms, and neuropathy. The nonspecific porphyria presentations, the complexity of heme biosynthesis, and difficulty in interpreting the laboratory tests make the diagnosis of porphyria challenging. Treatment of acute porphyria and avoidance of precipitating factors should be initiated early to prevent potentially severe long-term sequelae from nerve damage. Porphyric neuropathy is one such complication and is characterized by an axonal neuropathy with predominant motor involvement. Sensory neuropathy is also found but is less common. Although the exact pathophysiology of porphyric neuropathy remains uncertain, neural energy failure from heme deficiency and neurotoxicity from porphyrin precursors are probably the two main mechanisms. The understanding of porphyric neuropathy and manifestations of each type of porphyria along with timely implementation of appropriate tests can significantly assist in the diagnosis of these rare diseases.
\end{abstract}

Key words: porphyria; porphyric neuropathy; neuropathy; nervous system; heme

\section{INTRODUCTION}

The porphyrias are groups of uncommon diseases occurring secondary to an autosomal dominant inherited deficiency of enzymes in the heme biosynthetic pathway. Each porphyria is caused by a different enzymatic alteration that leads to accumulation of heme precursors, causing various clinical symptoms. Porphyrias have a high degree of symptom variance, including gastrointestinal, neurological, cutaneous,

Corresponding author: Doungporn Ruthirago, MD Contact Information: Doungporn.ruthirago@ttuhsc.edu DOI: $10.12746 /$ swrccc2016.0415.197 and psychiatric manifestations. ${ }^{1}$ Each presentation is nonspecific, mimicking other diseases that are much more common and making the diagnosis of porphyrias challenging. A high index of suspicion and appropriate laboratory investigation at the proper time are essential to establish the diagnosis.

Porphyrias can be broadly categorized into acute porphyrias and non-acute porphyrias depending on the presence of acute porphyric attacks. Patients who present with acute attacks need more urgent diagnosis and treatment to prevent potentially severe long-term sequelae. The well-known triad of 
abdominal pain, neuropathy,and psychiatric disturbances helps the clinician recognize acute porphyrias. ${ }^{2}$ However, in many cases patients present with only one or two symptoms. Sensory or motor neuropathy may be the most objective clinical manifestation that assists in the diagnosis of these uncommon diseases. Comprehensive reviews of the porphyrias are currently available in other literatures. ${ }^{3,4}$ The objective of this article is to simplify the understanding of the acute porphyrias and porphyric neuropathy.

The word "porphyria" is derived from the Greek word "porphura" which means purple. ${ }^{5}$ This is from the observation that the urine of porphyria patients has a red-purple color and becomes darker when exposed to light. Due to their enzymatic deficiency these patients have excess porphyrins and porphyrin precursors which accumulate in the body and are later excreted into urine and feces. ${ }^{6}$

\section{Pathophysiology}

\section{HEME BIOSYNTHESIS}

Heme is an oxygen carrier which is important to all aerobic reactions. It also functions as a source of electrons in the mitochondrial electron transport chain. Heme is synthesized in the bone marrow and liver and is required for the synthesis of hemoproteins-hemoglobin, myoglobin, and cytochromes, etc. Heme synthesis starts in the mitochondria where succinyl coenzyme A combines with glycine to form aminolevulinic acid (ALA). This process is catalyzed by aminolevulinic acid synthase (ALAS) which is the rate-limiting step of heme synthesis. Aminolevulinic acid synthase is inhibited by heme in a negative feedback pathway. When the requirement for heme increases or the reserves are depleted, ALAS is disinhibited and the heme metabolic pathway is activated. The next steps occur in the cytoplasm where two ALAs are converted to porphobilinogen (PBG) by aminolevulinic acid dehydratase (ALAD). Four PBGs are combined to form uroporphyrinogen by uroporphyrinogen cosynthase (UROS) enzyme. Uroporphyrinogen is later changed to coproporphyrinogen by the uroporphyrinogen decarboxylase (UROD) enzyme. Coproporphyrinogen enters the mitochondria where it is converted by the coproporphyrinogen oxidase (CPOX) enzyme to protoporphyrinogen. Protoporphyrinogen is changed into protoporphyrin IX by protoporphyrinogen oxidase (PPOX). The last step of synthesis is adding iron to protoporphyrin IX by the ferrochelatase $(\mathrm{FECH})$ enzyme to form heme (Table). ${ }^{7}$

Enzymatic defects in each step of the heme biosynthetic pathway cause susceptibility to different types of porphyria. The specific deficiency predicts which heme precursors or intermediates will accumulate and later be excreted in the feces or urine. These excess metabolites are sometimes oxidized into pigmented porphyrins and produce red/purple urine. ${ }^{1}$ Environmental factors also have an important role in development of acute porphyrias. Acute attacks occur from events that induce ALAS directly or increase the requirements of heme synthesis which indirectly disinhibit ALAS. ${ }^{8}$ For example, porphyrinogenic drugs, such as barbiturates and sulfa antibiotics, induce cytochrome P450, causing increased hepatic heme turnover. Infection and inflammation induce the expression of hepatic acute phase protein that catabolizes heme. ${ }^{3}$ Transcription of ALAS is stimulated by peroxisome proliferator-activated receptor gamma coactivator 1-alpha (PGC-1 $\alpha$ ), which involves energy metabolism and explains why acute porphyria is precipitated by starvation. ${ }^{9}$

\section{PATHOPHYSIOLOGY OF PORPHYRIC NEUROPATHY}

Every symptom of acute porphyrias can be explained by dysfunction of the nervous system. ${ }^{3} \mathrm{Ab}$ dominal pain during acute attacks reflects autonomic neuropathy that causes ileus, abdominal distension, followed by pain, constipation, nausea, and vomiting. Psychiatric disturbances can be explained by central nervous system involvement. Acute porphyric neurovisceral attacks develop in genetic-susceptible individuals when environmental triggers activate ALAS1, making deficient enzymes in later steps of heme biosynthesis become the rate-limiting step, leading to accumulation of porphyrin precursors, such as ALA and PBG. ${ }^{10}$

Porphyric neuropathy may be explained by 2 mechanisms. ${ }^{10}$ The first mechanism is direct neu- 


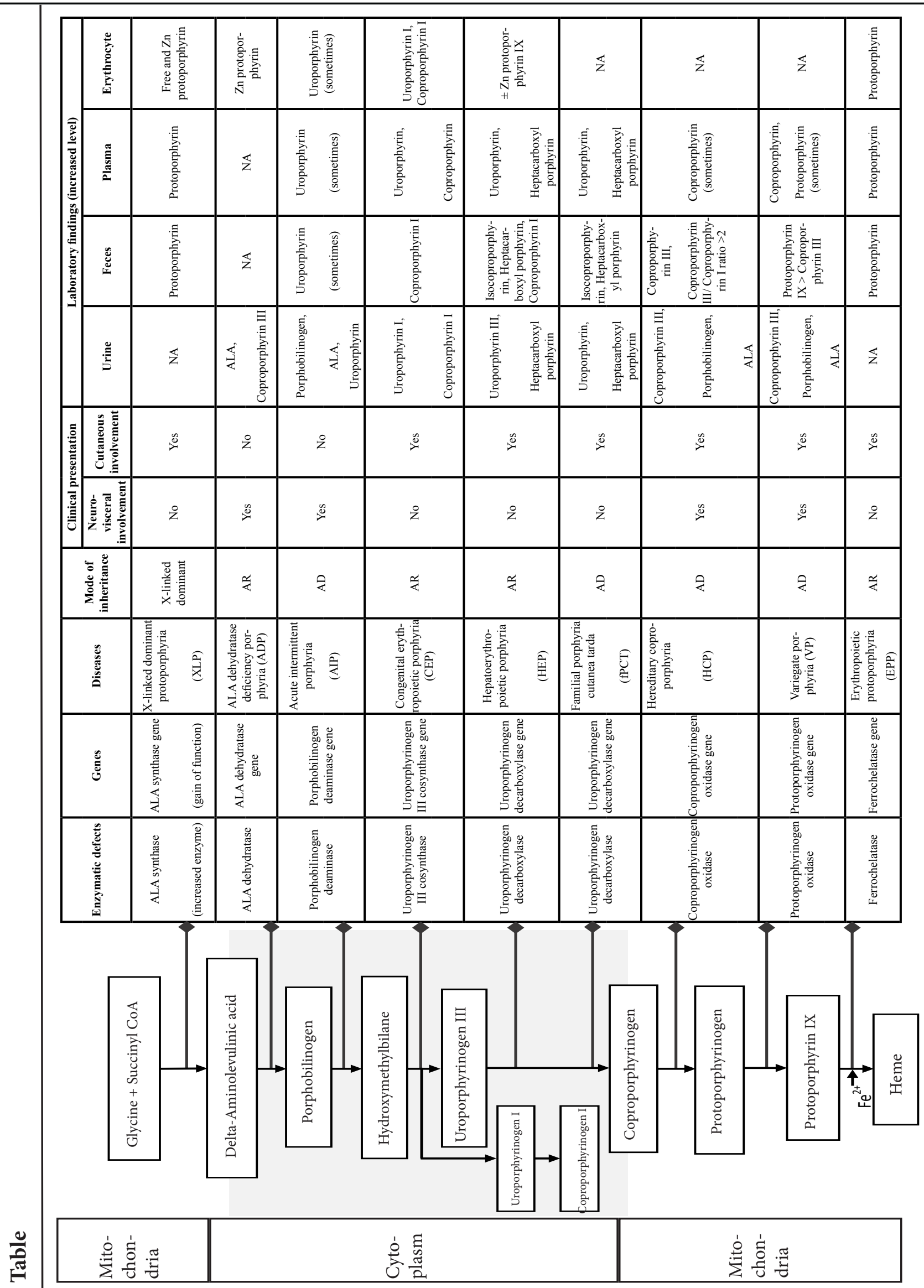


rotoxicity of accumulated porphyrin precursors, especially ALA. Aminolevulinic acid increases in acute attacks of porphyria and other porphyria-like neuropathies, such as lead poisoning and hereditary tyrosinemia. ${ }^{11}$ Aminolevulinic acid induces the formation of free radicals, causing oxidative damage to cell structures. ${ }^{12}$ The second mechanism is energy deficits due to inadequate heme synthesis, which is an essential component of electron transport system. Dysfunction of $\mathrm{Na}+/ \mathrm{K}+$ ATPase, which is energy-dependent, causes abnormal axon transport and neural dysfunction. ${ }^{13}$ Energy deficits also cause impaired detoxification systems in which cytochrome P450 fails to detoxify drugs and mitochondria cannot prevent oxidative damage (Figure). ${ }^{14}$

\section{Classifications}

The porphyrias may be classified based on the primary clinical manifestations as either acute or cutaneous or by the major site of the enzymatic defect as either hepatic or erythropoietic. ${ }^{1}$ This review will be based on porphyria classification by clinical manifestation and will mention nine types of porphyria according to the enzyme deficiency in each step of heme synthetic pathway with the exception of the Xlinked dominant protoporphyria which occurs from a gain of function mutation. ${ }^{3}$ Only four types of acute porphyrias are discussed in this review, and the five types of cutaneous porphyrias are listed at the end of the section. The Table shows the classification of porphyrias, related enzymatic deficiencies, gene mutations, mode of inheritance, clinical presentations, and laboratory finding.

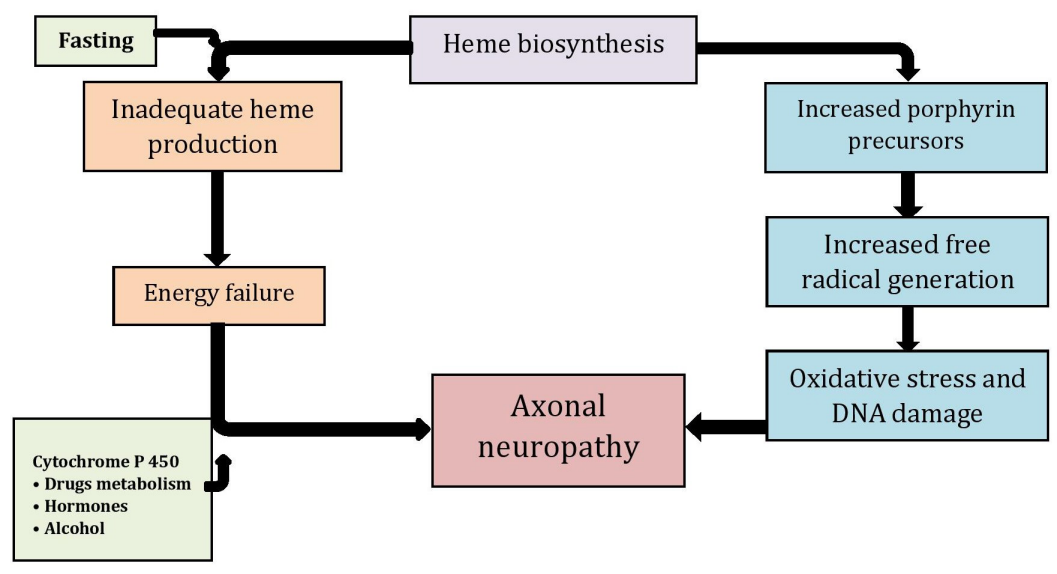

Figure-Schematic overview of the pathophysiology of porphyric neuropathy

Neuropathy occurs in $20-68 \%$ of porphyria patients. ${ }^{2}$ The typical pattern of porphyric neuropathy is predominantly motor axonal neuropathy. ${ }^{15,16}$ Abdominal pain usually starts days to weeks before neuropathy. The motor neuropathy is normally symmetrical and begins in proximal part of the upper extremities. However, the patterns of motor involvement are variable. ${ }^{10}$ Sensory neuropathy is less common, presents as neuropathic pain and distal limb paresthesia, and sometimes occurs in proximal distribution. ${ }^{2,17}$ Cranial nerve involvement can also occur but is infrequent.

\section{ACUTE PORPHYRIAS}

An acute life-threatening crisis or acute attacks are characteristic of acute porphyrias. Patients may present with prodromal symptoms, such as behavioral change, insomnia, anxiety, and later develop symptoms of acute attack. Acute attacks usually last no longer than 1-2 weeks before entering a recovery phase; they can be fatal if the patient develops severe neurological dysfunction. The acute attacks usually occur after puberty and rarely occur after menopause. Less than $10 \%$ of patients develop recurrent acute attacks. ${ }^{3}$ The mode of inheritance of acute porphyrias 
is autosomal dominant with incomplete penetrance except for aminolevulinic acid dehydratase deficiency porphyria which is an autosomal recessive disorder.

\section{Acute Intermittent Porphyria (AIP)}

Acute intermittent porphyria is the most common acute porphyria with an incidence of 1 in 20,000. ${ }^{1}$ It occurs due to a mutation in the porphobilinogen deaminase (PBGD) gene, which causes decreased PBGD enzyme activity (previously called hydroxymethylbilane synthase). Despite being inherited in an autosomal dominant pattern, AIP has variable penetrance which explains why some patients do not have a family history and why more than $90 \%$ of patients with an abnormal gene never develop an acute attack. Acute attacks occur in patients with genetic susceptibility who also have precipitating factors, such as hormones, drugs, or starvation. ${ }^{3}$

Acute attacks of AIP usually develop over two or more days with symptoms of abdominal pain, muscle weakness, neuropathy, sympathetic overactivity, hyponatremia, but no skin lesions. ${ }^{1}$ Urine tests during acute attacks demonstrate elevated urine porphobilinogen (PBG) which is sensitive and specific for acute porphyrias. Low PBGD enzyme activity in red blood cells confirms the diagnosis of AIP; however, some patients may not have an enzyme deficiency. The gold standard for diagnosis of AIP is genetic testing for specific mutations. ${ }^{1,3}$

\section{Hereditary Coproporphyria (HCP)}

Patients with HCP present with classic neurovisceral symptoms of acute attack. In some cases cutaneous photosensitivity is also present associated with blistering skin lesions similar to those in porphyria cutanea tarda (PCT). Hereditary coproporphyria occurs from a mutation in the coproporphyrinogen oxidase (CPOX) gene, causing decreased CPOX enzyme activity. Laboratory tests during acute attacks show elevated urine delta-ALA and PBG. Fecal coproporphyrin in appropriate ratio is sensitive for diagnosis of HCP. In asymptomatic carriers, urine and feces have elevated coproporphyrins, especially type III. However, isolated excess urine coproporphyrin is nonspecific as it can be found in liver diseases. Genetic testing for the CPOX mutation should be performed to confirm the diagnosis of HCP in both symptomatic patients and asymptomatic family members. ${ }^{1}$

\section{Variegate Porphyria (VP)}

Variegate porphyria, similar to HCP, presents with neurovisceral symptoms similar to other acute porphyrias though frequently less severe. Patients may present with cutaneous photosensitivity developing blistering skin lesions more frequently than HCP patients. The incidence of VP is highest in South Africa. It occurs from a mutation of the protoporphyrinogen oxidase (PPOX) gene, causing decreased PPOX enzyme activity. Urine delta-ALA and PBG are elevated during acute attacks but usually are normal between attacks. Plasma porphyrins are increased during attacks. In asymptomatic carriers, urine tests show elevated coproporphyrin, and fecal tests show excess coproporphyrin and protoporphyrin. Genetic testing should be performed to confirm the diagnosis. ${ }^{1}$

\section{Aminolevulinic aCid dehydRatase deficiency PORPHY- RIA (ADP)}

Aminolevulinic acid dehydratase deficiency porphyria is the only acute porphyria that is transmitted in an autosomal recessive pattern. It is extremely rare with less than 10 cases reported worldwide. It occurs from a mutation of the aminolevulinic acid dehydratase (ALAD) gene, causing decreased ALAD enzyme activity. Patients present with symptoms of acute attack similar to AIP. Skin lesions are not present. Laboratory tests during acute attacks show elevated urine ALA but normal PBG. ${ }^{1}$

\section{Cutaneous PORPhyRIAS}

5. Sporadic Porphyria cutanea tarda and familial porphyria cutanea tarda (sPCT and fPCT)

6. Erythropoietic protoporphyria (EPP)

7. X-linked dominant protoporphyria (XLP)

8. Congenital erythropoietic porphyria (CEP)

9. Hepatoerythropoietic porphyria (HEP)

The details of cutaneous porphyrias are beyond the 
scope of this review. However, the summary of their enzymatic defects, mutations, modes of inheritance, clinical presentations, and laboratory findings are also shown in the Table.

\section{Clinical manifEstations}

The presentation of the acute porphyrias is variable and nonspecific; patients usually develop symptoms in several systems.

\section{VISCERAL MANIFESTATIONS}

Abdominal pain is the most common and most troublesome presenting symptom of acute porphyria with an occurrence rate of $85-95 \% .{ }^{18}$ Patients usually complain of constant, poorly localized pain associated with nausea, vomiting, abdominal distension, and constipation that mimics an acute abdomen. Fever and leukocytosis rarely occur since the pain is neuropathic and not inflammatory. Bladder dysfunction can also develop with urinary retention, incontinence, and dysuria. ${ }^{19}$

\section{NeUROLogical manifESTATIONS}

Central nervous system symptoms, such as seizure and encephalopathy, are reported in 5-30\% and $2-10 \%$ of patients, respectively. Seizure in acute porphyrias may be triggered by hyponatremia and hypomagnesemia. ${ }^{10,20}$ In acute attacks associated with encephalopathy, abnormal magnetic resonance imaging similar to that found in posterior reversible encephalopathy syndrome (PRES) has been reported. ${ }^{21}$ Peripheral neuropathy can present as weakness, sensory disturbances, pain, and respiratory muscle weakness in $20-68 \%, 7-38 \%, 20-70 \%$, and $9-20 \%$, respectively. 2,10 Motor symptoms are more common than sensory symptoms. Autonomic dysfunction also occurs commonly, presenting as tachycardia, hypertension, restlessness, tremor, and sweating.

\section{Psychiatric manifestations}

Psychiatric symptoms are reported in 20$30 \%$ of patients during acute attacks. ${ }^{22}$ These include anxiety, depression, insomnia, restlessness, disori- entation, hallucinations, and paranoia. Chronic pain and depression may also be present in some patients after frequent exacerbations and are associated with increased risk of suicide.

\section{Cutaneous manifestations}

Photosensitivity presenting as redness, pain, swelling in sun-exposed areas, and bullous lesions occurs in several types of porphyrias. ${ }^{1}$ Cutaneous lesions were reported in $60 \%$ of patients with variegate porphyria and $5 \%$ of hereditary coproporphyria but are not found in acute intermittent porphyria. ${ }^{23}$

\section{Other MANIFESTATIONS}

Hyponatremia occurs in $30 \%$ of patients, sometimes due to the syndrome of inappropriate antidiuretic hormone secretion but also due to excessive gastrointestinal loss from vomiting, poor oral intake, and excess renal losses. ${ }^{18}$ Dark or red urine may also be an early symptom, but it does not always occur during acute attacks. If present, it suggests the diagnosis. Patients with porphyrias also have an increased incidence of hypertension and chronic kidney disease and have an increased risk for chronic liver disease and hepatocellular carcinoma. ${ }^{17}$

\section{Diagnosis}

Porphyrias usually present with nonspecific symptoms. Therefore, laboratory tests are essential to confirm or exclude the diagnosis. Common investigations in acute porphyrias include biochemical tests to measure porphyrins and porphyrin precursors, to detect enzyme activities, and to confirm the diagnosis with DNA tests. For patients who present with neuropathy, electrophysiological tests assist in differentiating the type of neuropathy. Pathological studies are not usually required for diagnosis but help improve the understanding of porphyric neuropathy.

\section{BIOCHEMICAL TESTS}

Deficiency of enzymes in each type of porphyrias causes accumulation of different precursors and intermediates. During acute attacks, these excess 
substances accumulate in the liver or bone marrow and later enter the blood. The water-soluble intermediates are excreted in the urine, while the water-insoluble intermediates are excreted in the feces. ${ }^{1}$ Porphyrins are named according to the number of carboxyl groups as octacarboxyl porphyrin (uroporphyrin), heptacarboxyl porphyrin, hexacarboxyl porphyrin, pentacarboxyl porphyrin, tetracarboxyl porphyrin (coproporphyrin), tricarboxyl prophyrin (harderoporphyrin), dicarboxyl porphyrin (protoporphyrin). The more carboxyl groups present, the more water-soluble is the compound. Tricarboxyl and dicarboxyl prophyrins are excreted in bile and feces, while coproporphyrin are excreted in urine and feces. ${ }^{24}$ Porphyrin precursors, such as ALA and PBG, are elevated in all types of acute porphyrias, except for ADP which has isolated ALA elevation.

During acute attacks, the first-line tests with high sensitivity and specificity are measuring urinary ALA, PBG, and total porphyrins. If all the levels are normal, acute porphyrias can be excluded. If the levels are increased more than 5 times normal, acute porphyria is highly possible, and second-line tests should be done to differentiate the type of porphyria. Nonspecific elevation of less than three times can be found in dehydration. ${ }^{1}$ However, if the urine level is done after the onset of acute attack, ALA and PBG levels can be normal. Urine porphyrins may remain elevated longer than the precursors, but they are less specific for porphyrias. Measurement of individual porphyrins in plasma, urine, and feces can be done to help differentiate the type of porphyrias by using high performance liquid chromatography (HPLC). ${ }^{1,24} \mathrm{How}-$ ever, the patterns of elevation are difficult to interpret and other conditions can cause elevation of porphyrins. For example, liver diseases, some bone marrow diseases and lead poisoning can cause increase in total urine porphyrin and coproporphyrin. This pattern is also found in hereditary coproporphyria and in variegate porphyria. ${ }^{7}$

For cutaneous porphyrias and acute porphyrias with cutaneous manifestations, such as hereditary coproporphyria and variegate porphyria, measurement of plasma porphyrins when the patient presents with skin symptoms can be used as a first-line test to screen for porphyria. ${ }^{1}$ If total plasma porphyrin is increased, further tests should be done to confirm the diagnosis.

Measurement of enzyme activity in erythrocytes (PBGD, ALAD) and lymphocytes (CPOX and PPOX) can help to support diagnosis of AIP, ADP, $\mathrm{HCP}$, and VP, respectively. ${ }^{3}$ However, there is some overlap between the normal range and the spectrum of porphyrias, and some mutations do not cause deficiency in blood cells. It is recommended that an enzyme activity assay be used to detect carriers in the family when a deficiency in the index case is already confirmed. ${ }^{1}$

\section{Genetic tests}

The gold standard test to confirm diagnosis of porphyria is DNA testing to identify specific mutations. It can also be used to detect carriers of gene mutation in families after the mutation is identified in the index case. DNA testing can detect more than $97 \%$ of disease-causing mutations, especially with significantly elevated PBG levels. ${ }^{1}$ Therefore, porphyrin precursors, such as ALA and PBG, and biochemical testing in plasma, urine, and feces should be done before requesting a DNA test. DNA tests from blood samples can be done either during acute attacks or in asymptomatic phases. In patients who present with acute attacks without skin lesions, the "Triple test" can be requested to detect mutations for AIP, VP, and HCP. ${ }^{1}$, 25,26

\section{ELECTROPHYSIOLOGICAL FINDINGS}

As porphyric neuropathy is fundamentally an axonal neuropathy with predominant motor nerve dysfunction, electrodiagnostic findings typically support this pattern of neuropathy. Nerve conduction studies (NCS) during acute attacks show reductions in compound motor unit action potential (CMAP) with relatively preserved conduction velocities. Conduction abnormalities of sensory nerves are sometimes demonstrated but are less common than motor nerves. ${ }^{27}$, 28 In later stages, when muscle weakness is more prominent, NCS may show progressive reductions in CMAP amplitudes. Electromyography (EMG) demon- 
strates wide spread fibrillation potentials compatible with denervation, especially in proximal muscles. In later stages, EMG may show polyphasic motor unit action potentials with higher duration and amplitude, predicting denervation and re-innervation patterns. ${ }^{29}$ Between acute attacks, even without clinical neuropathy, NCS may show reduced inward rectification $\left(I_{H}\right)$, which indicates subclinical dysfunction of axonal metabolism. ${ }^{15}$ During acute attacks, axon excitability recordings show depolarization of axonal membrane secondary to impairment of $\mathrm{Na}+/ \mathrm{K}+$ ATPase function. $^{10,15,30}$ Appropriate treatment in early phase of porphyric attack can resolve these neuropathic patterns. However, if the treatment is delayed or if the attack is severe, neuropathy may persist and only partially improve over time..$^{15}$

The clinical manifestations of porphyric neuropathy can overlap with Guillain-Barre syndrome (GBS). The electrophysiological patterns of axonal neuropathy, such as reduced amplitudes, relative preservation of $\mathrm{H}$ reflexes, $\mathrm{F}$ waves and distal latency, minimal conduction slowing, and absence of conduction block, may help differentiate it from demyelinating patterns typically found in GBS. ${ }^{2,31}$ However, differentiating porphyric neuropathy from the axonal type of GBS is difficult and may need serial electrodiagnostic studies and other laboratory tests.

\section{Pathological findings}

Pathological studies demonstrate severe denervation of motor nerves and central chromatolysis of anterior horn cells, suggestive of dying-back Wallerian degeneration, with relatively spared sensory nerves. Muscle biopsy shows significant loss of nerve fibers compatible with neurogenic change and limited changes of muscle denervation atrophy. ${ }^{27,} 28,32$

The proximal predominant pattern of porphyric neuropathy may be explained by retrograde axonal transport of neurotoxic substances, such as ALA, from the distal part of the axon to motor neuron. Heme precursors may enter at neuromuscular junctions which do not have a blood-nerve barrier and are transported initially to neurons innervating proximal muscles, causing proximal more than distal weakness. ${ }^{2,33}$

\section{TREATMENT}

Most patients who present with acute attacks of porphyrias require hospitalization for pain control, hydration, and treatment of nausea and vomiting. Treatments can be broadly divided into symptomatic treatment, specific treatment of porphyrias, and counseling.

\section{SYMPTOMATIC TREATMENT}

Abdominal and limb pain can be controlled with acetaminophen, nonsteroidal anti-inflammatory drugs, and opiates as needed. Nausea and vomiting can be treated with promethazine, ondansetron, or other antiemetic drugs. Maintaining water and electrolyte balance is very important. Adequate hydration is needed, as well as preventing hyponatremia which can provoke seizures. Constipation can be treated with bulk laxatives or lactulose. Some patients who have hypertension and tachycardia from sympathetic overactivity may need $\beta$-blockers, such as propranolol and atenolol. ${ }^{3}$ Physical and speech therapy are helpful for patients who develop muscle weakness or bulbar involvement. Some patients with respiratory muscle weakness may need mechanical ventilation. Close monitoring in the intensive care unit is needed in patients with severe acute attacks. Insomnia and anxiety can be managed with benzodiazepines. Antipsychotic medications are sometimes required for psychotic symptoms. ${ }^{3,4}$

\section{SPECIFIC TREATMENT OF ACUTE PORPHYRIAS}

Glucose inhibits ALAS and decreases porphyrin synthesis in the liver. ${ }^{34}$ Adequate carbohydrate and calorie intake is important to suppress disease activity and accelerate recovery. Intravenous glucose should be administered if the patients cannot tolerate oral diets. Intravenous heme (or hemin) $4 \mathrm{mg} / \mathrm{kg} /$ day, given daily for 4-14 days, is a specific and effective treatment if started early before severe nerve damage occurs. ${ }^{35}$ It replaces heme deficiency in the liver and suppresses production of porphyrin precursors. Some porphyria experts recommend starting hemin if symptoms do not improve within 1-2 days of intravenous glucose. ${ }^{1}$ It should be started in patients 
who have elevated urinary PBG levels. Delayed treatment can lead to nerve damage, chronic pain, and muscle weakness. ${ }^{10}$ Hemin has some side effects, such as phlebitis and coagulopathy, which could be prevented by dilution with human albumin and using a central line or large peripheral vein. Less than $10 \%$ of patients with recurrent acute attacks need preventive treatment with intravenous hemin and rarely liver transplantation. ${ }^{1,3}$

\section{Counseling}

Counseling is one of the most important management steps in acute porphyrias. Patients should be advised to avoid precipitating factors. Alcohol and porphyrinogenic drugs, such as barbiturates, sulfonamide antibiotics, hormonal substances, some antiepileptic medications, should be avoided. Infections should be treated. ${ }^{1}$ The patients and their physicians should check with reliable sources regarding the safety of drugs before initiating any new treatment. Adequate carbohydrate and calorie intake is important. Porphyria patients should be educated to avoid starvation. A dietitian should be consulted if weight loss is truly necessary. ${ }^{1,3}$ Genetic counseling for the patient and their relatives is another important issue. Children of patients with acute porphyrias have a $50 \%$ chance of acquiring an abnormal gene. Genetic testing for relatives to detect heterozygous carriers and educating them to avoid precipitating factors can prevent acute attacks.

After specific treatment, the symptoms of acute attacks are usually abolished. Levels of ALA and PBG excreted in the urine return to normal. Prognosis of porphyrias is generally good if the treatments are initiated early and the precipitating factors are removed before severe nerve damage has developed. On the other hand, with delayed treatment, patients may develop nerve damage that results in chronic pain and muscle weakness for several months or an incomplete recovery. ${ }^{10}$

\section{Conclusions}

Porphyrias are rare inherited metabolic disorders that present with neurovisceral, cutaneous, and psychiatric symptoms. Acute porphyrias occur from environmental triggers in the presence of genetic susceptibility, leading to accumulation of porphyrins and their precursors. Porphyric neuropathy is believed to develop from conditions that cause relative heme deficiency and neurotoxicity from porphyrin precursors. The typical electrophysiological pattern of porphyric neuropathy is axonal neuropathy with predominant motor involvement. The timely diagnosis and early initiation of specific treatment are important to prevent severe porphyric neuropathy and its sequelae. Symptomatic treatment during the attacks and counseling about carrier detection and avoidance of precipitating factors should be provided to the patients.

Author Affiliation: Doungporn Ruthirago and Parunyou Julayanont are residents in the Department of Neurology at Texas Tech University Health Sciences Center in Lubbock, TX. Supannee Rassameehiran is a resident in the Department of Internal Medicine at TTUHSC in Lubbock, TX. Received: 04/19/2016

Accepted: 06/14/2016

Reviewers: Catherine Jones MD

Published electronically: 07/15/2015

Conflict of Interest Disclosures: None

\section{REFERENCES}

1. The American Porphyria Foundation. http://www.porphyriafoundation.com; 2015 [accessed 23.10.15].

2. Albers JW, Fink JK. Porphyric neuropathy. Muscle Nerve 2004;30:410-422.

3. Puy H, Gouya L, Deybach JC. Porphyrias. Lancet. 2010;375:924-937.

4. Balwani M, Desnick RJ. The porphyrias: advances in diagnosis and treatment. Blood 2012;120:4496-4504.

5. Hoppe-Seyler F. hematoporphyrin. Medizinich-Chemische Untersuchungen 1871;4:531-539.

6. Baumstark F. Zwei pathologische harnfarbstoff. Pfugers Arch Ges Physiol 1874;9:568-584.

7. Albers J. Porphyria neuropathy. In: Mendell J, Kissel J, Cornblath D, eds. Diagnosis and management of peripheral nerve disorders. New York: Oxford University Press; 2001:344-366.

8. Meyer UA, Schuurmans MM, Lindberg RL. Acute por- 
phyrias: pathogenesis of neurological manifestations. Semin Liver Dis 1998;18:43-52.

9. Handschin C, Lin J, Rhee J, et al. Nutritional regulation of hepatic heme biosynthesis and porphyria through PGC1alpha. Cell 2005;122:505-515.

10. Lin CS, Lee MJ, Park SB, et al. Purple pigments: the pathophysiology of acute porphyric neuropathy. Clin Neurophysiol 2011;122:2336-2344.

11. Egger N, Lee C, Anderson K, et al. Disorders of heme biosynthesis. In: Fernandes J, Saudubray J, Vanden Berghe $\mathrm{G}$, eds. Inborn metabolic diseases: diagnosis and treatment. Heidelberg: Springer; 2006.

12. Moore M. The pathogenesis of acute porphyria. Aspects Med 1990;11:49-57.

13. Sengupta A, Hon T, Zhang L. Heme deficiency suppresses the expression of key neuronal genes and causes neuronal cell death. Brain Res Mol Brain Res 2005;137:23-30.

14. Thunell S, Harper P, Brock A, et al. Porphyrins, porphyrin metabolism and porphyrias. II. Diagnosis and monitoring in the acute porphyrias. Scand J Clin Lab Invest 2000;60:541-559.

15. Lin CS, Krishnan AV, Lee MJ, et al. Nerve function and dysfunction in acute intermittent porphyria. Brain 2008;131:2510-2519.

16. Wu CL, Ro LS, Jung SM, et al. Clinical presentation and electrophysiological findings of porphyric neuropathies: a follow-up study. Muscle Nerve 2015;51:363-369.

17. Ridley A. The neuropathy of acute intermittent porphyria. Q J Med 1969;38:307-333.

18. Anderson KE, Bloomer JR, Bonkovsky HL, et al. Recommendations for the diagnosis and treatment of the acute porphyrias. Ann Intern Med 2005;142:439-450.

19. Trier H, Krishnasamy VP, Kasi PM, et al. Clinical manifestations and diagnostic challenges in acute porphyrias. Case Rep Hematol. 2013;2013:628602.

20. Bylesjö I, Forsgren L, Lithner F, et al. Epidemiology and clinical characteristics of seizures in patients with acute intermittent porphyria. Epilepsia 1996;37:230-235.

21. Utz N, Kinkel B, Hedde JP, et al. MR imaging of acute intermittent porphyria mimicking reversible posterior leukoencephalopathy syndrome. Neuroradiology 2001;43:10591062.

22. Crimlisk HL. The little imitator--porphyria: a neuropsychiatric disorder. J Neurol Neurosurg Psychiatry 1997;62:319-328.

23. Anderson K, Sassa S, Bishop D. Disorders of heme biosynthesis: X-linked sideroblastic anemia and the porphyrias. In: CR S, Beaudet A, Sly W, eds. The metabolic basis of inherited disease. 8 ed. New York: McGraw-Hill; 2001.

24. Tests for Porphyria diagnosis: American Porphyria Foundation. Vol 2015.

25. Nordmann Y, de Verneuil H, Deybach JC, et al. Molecu- lar genetics of porphyrias. Ann Med 1990;22:387-391.

26. Desnick R, Astrin K, Anderson K. Inherited porphyrias. In: Rimoin D, Connor J, Pyeritz R, eds. Emery and Rimoin's principles and practice of medical genetics. New York: Churchill Livingstone; 2002:2587-2623.

27. Maytham DV, Eales L. Electrodiagnostic findins in porphyria. S Afr Med J. 1971:99-100.

28. Flügel KA, Druschky KF. Electromyogram and nerve conduction in patients with acute intermittent porphyria. J Neurol 1977;214:267-279.

29. Albers JW, Robertson WC, Daube JR. Electrodiagnostic findings in acute porphyric neuropathy. Muscle Nerve 1978;1:292-296.

30. Kaji R, Sumner AJ. Ouabain reverses conduction disturbances in single demyelinated nerve fibers. Neurology 1989;39:1364-1368.

31. Elder GH, Hift RJ, Meissner PN. The acute porphyrias. Lancet 1997;349:1613-1617.

32. Cavanagh JB, Mellick RS. On the Nature of the Peripheral Nerve lesions associated with acute intermittent porphyria. J Neurol Neurosurg Psychiatry 1965;28:320-327.

33. Anonymous. Case records of the Massachusetts General Hospital. Weekly clinicopathological exercises. Case 39-1984. N Eng J Med 1984;311:839-847.

34. Windebank A, Bonkovsky H. Porphyric neuropathy. In: Dyck P, Thomas P, Griffin J, eds. Peripheral neuropathy. Philadelphia: Suanders; 1993:1161-1168.

35. Laiwah AC, McColl KE. Management of attacks of acute porphyria. Drugs 1987;34:604-616. 